\title{
Happiness, Meaning, and Psychological Richness
}

\author{
Shigehiro Oishi ${ }^{1} \cdot$ Hyewon $\mathrm{Choi}^{2} \cdot$ Minkyung Koo $^{3} \cdot$ Iolanda Galinha $^{4} \cdot$ Keiko Ishii $^{5} \cdot$ Asuka Komiya $^{6}$. \\ Maike Luhmann ${ }^{7}$. Christie Scollon ${ }^{8}$. Ji-eun Shin ${ }^{9} \cdot$ Hwaryung Lee $^{10}$. Eunkook M. Suh ${ }^{10}$. Joar Vitters $\emptyset^{11}$. \\ Samantha J. Heintzelman ${ }^{12} \cdot$ Kostadin Kushlev $^{13}$ • Erin C. Westgate ${ }^{14} \cdot$ Nicholas Buttrick $^{2}$ - Jane Tucker ${ }^{15}$. \\ Charles R. Ebersole ${ }^{2}$. Jordan Axt ${ }^{16}$. Elizabeth Gilbert ${ }^{17} \cdot{\text { Brandon W. } \mathrm{Ng}^{18}}^{18}$ Jaime Kurtz ${ }^{19} \cdot$ Lorraine L. Besser $^{20}$
}

Published online: 23 June 2020

(C) The Society for Affective Science 2020

\begin{abstract}
What kind of life do people want? In psychology, a good life has typically been conceptualized in terms of either hedonic or eudaimonic well-being. We propose that psychological richness is another neglected aspect of what people consider a good life. In study 1 (9-nation cross-cultural study), we asked participants whether they ideally wanted a happy, a meaningful, or a psychologically rich life. Roughly 7 to $17 \%$ of participants chose the psychologically rich life. In study 2, we asked 1611 Americans and 680 Koreans what they regret most in their lives; then, if they could undo or reverse the regretful event, whether their lives would have been happier, more meaningful, or psychologically richer as a result. Roughly $28 \%$ of Americans and $35 \%$ of Koreans reported their lives would have been psychologically richer. Together, this work provides a foundation for the study of psychological richness as another dimension of a good life.
\end{abstract}

Keywords Happiness $\cdot$ Meaning in life $\cdot$ Psychological richness

What kind of life do people want? Many psychologists conceptualize well-being in terms of hedonic (e.g., Diener, Suh, Lucas, \& Smith, 1999) or eudaimonic well-being (e.g., Ryff,

Handling editor: Phoebe Ellsworth

Our studies were not preregistered.

Electronic supplementary material The online version of this article (https://doi.org/10.1007/s42761-020-00011-z) contains supplementary material, which is available to authorized users.

\section{Shigehiro Oishi}

soishi@ virginia.edu

1 Department of Psychology, University of Virginia, 10027 Charlottesville, VA, USA

2 University of Virginia, Charlottesville, VA, USA

3 University of Illinois at Urbana Champaign, Champaign, IL, USA

4 Universidade Autónoma de Lisboa, Lisbon, Portugal

5 Nagoya University, Nagoya, Japan

6 Hiroshima University, Hiroshima, Japan

7 Ruhr University Bochum, Bochum, Germany

8 Western Washington University, Bellingham, WA, USA

9 Chonnam National University, Gwangju, South Korea
1989). A happy life, which maps onto hedonic well-being, is best characterized by pleasantness, comfort, safety, and stability. People who say they have a happy life tend to have

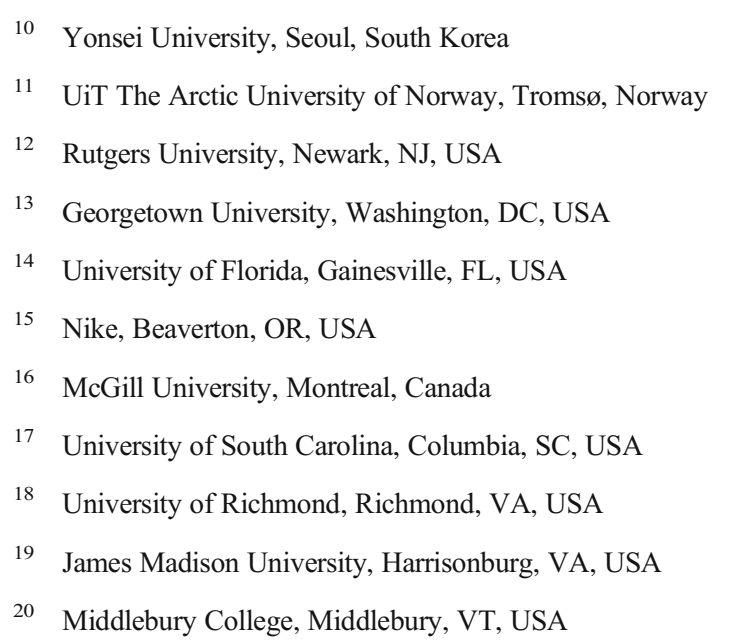


material and relational wealth (Diener et al., 1999). It is clear that people desire a happy life; $69 \%$ of respondents from 42 countries rated happiness as "extremely important" (Diener, 2000). Another widely accepted ideal is a meaningful life, which maps onto eudaimonic well-being. It is best characterized by a life with purpose, meaning, devotion, service, and sacrifice. It too is desirable, with most people reporting their lives are pretty meaningful (Heintzelman \& King, 2014).

But are a happy life and a meaningful life the only options? In this paper, we examine a third possibility: the psychologically rich life (see Besser \& Oishi, in press for theoretical discussions). A psychologically rich life is best characterized by variety, novelty, and interest. One problem with happy and meaningful lives is that both can be monotonous and repetitive. A person with a steady - perhaps even rewarding - 9-to5 job, happily married with children, may have a happy and, in many respects, meaningful life, but not necessarily a life rich in diverse experiences. Recent studies show that people dislike the state of boredom so much so that some are even willing to give themselves a painful electric shock rather than sit idly (Westgate \& Wilson, 2018). In other words, a happy or meaningful life can also be boring. In contrast, psychological richness fulfills the need for complex, varied experiences, of the sort that change people's view of the world and their place in it.

Is a psychologically rich life distinct from a happy or meaningful one? Recent studies suggest that it is. While psychological richness is correlated with happiness and meaning in life, structural equation models find that a tripartite model of wellbeing — with richness, happiness, and meaning as three distinct latent constructs - best fits people's self-reports (Oishi, Choi, Heintzelman, et al., 2020). Likewise, psychological richness does not appear to simply reflect a eudaimonic life: self-reports of psychological richness are actually less strongly associated with Ryff's (1989) 6 facets of psychological wellbeing than happiness or meaning in life (Oishi, Choi, Heintzelman, et al., 2020).

Moreover, empirical evidence supports the claim that psychological richness is uniquely related to novel, complex, (and sometimes negative) perspective-changing events in ways that happiness and meaning are not. For instance, psychological richness increased over the course of the semester among students studying abroad, whereas it did not change among students studying on campus (Oishi, Choi, Liu, \& Kurtz, 2020); there were no parallel changes in life satisfaction or meaning in life. The emotional correlates of psychological richness also appear distinct: people high in psychological richness report experiencing both positive and negative emotions more intensely, whereas those high in happiness and meaning report experiencing positive emotions more intensely but negative emotions less intensely (Oishi, Choi, Heintzelman, et al., 2020). Likewise, experimental manipulations of perspective change reliably increase psychological richness (Oishi \&
Choi, 2020) but not perceived meaning (Westgate \& Losee, 2020).

Finally, psychological richness appears uniquely related to openness and curiosity. For instance, using the multi-method multi-trait approach, openness to experience was a consistent, strong predictor of a psychologically rich life, but not life satisfaction or meaning in life (Oishi et al., 2019). Similarly, people high in happiness and meaning were more politically conservative and system justifying, whereas those high in psychological richness tend to be politically liberal and do not system justify (Oishi, Westgate, Heintzelman, et al., 2020). Finally, psychological richness predicts curiosity, above and beyond happiness and meaning, whereas happiness and meaning do not.

In sum, recent studies show that a psychologically rich life is related to but distinct from a happy life and a meaningful life. But is it a life people want? Whereas happiness and meaning appear to have universal appeal, psychological richness may involve negative aspects, including potential risk or danger. Thus, it is not clear whether people actually want or value a psychologically rich life more so than a happy or meaningful one. The value people place on emotions, and what they believe about them, has important implications for emotion regulation and experiences (e.g., Ford, Lwi, Hankin, Gentzler, \& Mauss, 2018; Gruber, Mauss, \& Tamir, 2011; Tsai, 2007). Thus, understanding the kinds of lives people want sheds new light on their values associated with emotional wellbeing.

To explore this question, we conducted 2 studies investigating the degree to which people value a psychologically rich life, relative to a happy or meaningful life. In study 1, participants living in 9 diverse countries were explicitly asked the degree to which they value a psychologically rich life, a happy life, and a meaningful life. In study 2, participants in the USA and Korea were indirectly asked the degree to which they value these three types of a good life. Together, the current research provides important descriptive information regarding people's ideal lives.

\section{Study 1: a 9-Nation Study}

\section{Method}

\section{Participants}

A total of 3728 participants participated in this study from 13 samples in 9 nations: USA, Japan, Korea, India, Norway, Singapore, Portugal, Germany, and Angola. We attempted to have a broad range of samples so as to maximize generalizability of our findings (Henrich, Heine, \& Norenzayan, 2010). In the first three nations, we recruited multiple samples: two college samples and one non-college sample from 
Amazon Mechanical Turk (Mturk) in the USA, two college samples in Japan, and one college sample and one panel sample in Korea. In India, we recruited a non-college sample from Mturk, and in Norway, we recruited a combination of college and non-college participants from a college or Facebook. In Singapore, Portugal, Germany, and Angola, we recruited participants from a single college in each nation. Demographic information and sample size for all countries is presented in Table 1. Data collection took place between July 2017 and October 2018.

For the two Mturk samples (USA, India), we included attention check items in which participants were instructed to choose "quite a bit (4)" on a 5-point scale ( $1=$ not at all, $5=a$ great deal) for all four items asking their attitudes toward the US economy regardless of their real opinions. Participants who chose other options (i.e., 1, 2, 3, or 5) in any of the four items indicated they were not paying attention to the survey and were excluded from the analyses. Out of 1007 participants, 119 (11.8\%) failed the attention check in the US Mturk sample, whereas 449 participants (44.8\%) out of 1002 failed in the Indian Mturk sample. Thus, the final sample across all nine countries consisted of 3160 participants. All participants received either partial course credit or payment as compensation.

\section{Procedures and Materials}

Participants were asked to describe, in their own words, the ideal life they would like to lead. Then, they were asked to indicate the degree to which the ideal life they had just described was characterized by 15 terms: "stable," "comfortable," "simple," "happy," "pleasant," "eventful," "dramatic," "interesting," "full of surprise," "psychologically rich," "meaningful," "fulfilling," "virtuous," "sense of purpose," and "involves devotion" on a 7-point scale $(1=$ not at all, $7=$ very $m u$ ch $)$. The first five items were intended to capture characteristics of a happy life, the next five items a psychologically rich life, and the last five items a meaningful life. Finally, we asked participants to indicate which one of the three lives they would like to lead, if they had to choose only one: a happy life, a meaningful life, or a psychologically rich life.

Participants also completed current levels of hedonic wellbeing and eudaimonic well-being. For hedonic well-being, life satisfaction, positive affect, and negative affect were measured. Life satisfaction was assessed with the Satisfaction with Life Scale (Diener, Emmons, Larsen, \& Griffin, 1985; mean Cronbach's $\alpha=.84)$ using a 7-point scale $(1=$ strongly disagree, $7=$ strongly agree). Positive affect and negative affect were assessed with the Scale of Positive and Negative Experience (Diener et al., 2010; mean Cronbach's $\alpha$ for positive affect $=.90$; mean Cronbach's $\alpha$ for negative affect $=$ .83) using a 5-point scale ( $1=$ very rarely or never, $5=$ very often or always). For eudaimonic well-being, presence of meaning in life, a subscale of the Meaning in Life Questionnaire (Steger, Frazier, Oishi, \& Kaler, 2006; mean Cronbach's $\alpha=.86$ ), was measured using a 7-point scale (1 $=$ absolutely untrue, $7=$ absolutely true) .

\section{Results}

\section{What Is an Ideal Life? Self-Rated Characteristics}

We first analyzed how participants characterized the good life they imagined using their own self-ratings ("The ideal life that I described is..." "stable," "comfortable," etc.). First, given the diverse samples, we checked for cross-cultural measurement equivalence of the 3 -factor structure of the good life.
Table 1 Demographic information for participants in each sample in study 1

\begin{tabular}{|c|c|c|c|c|c|c|c|}
\hline \multirow[t]{2}{*}{ Nation } & \multirow[t]{2}{*}{ Sample } & \multirow[t]{2}{*}{$n$} & \multicolumn{3}{|c|}{ Gender } & \multicolumn{2}{|l|}{ Age } \\
\hline & & & Male & Female & Other or unspecified & Mean $(S D)$ & Range \\
\hline \multirow[t]{3}{*}{ USA } & College 1 & 204 & 47 & 149 & 8 & $18.30(0.80)$ & $17-21$ \\
\hline & College 2 & 199 & 92 & 105 & 2 & $18.93(1.04)$ & $17-23$ \\
\hline & Mturk & 888 & 372 & 514 & 2 & $38.59(12.46)$ & $18-76$ \\
\hline \multirow[t]{2}{*}{ Japan } & College 1 & 119 & 45 & 74 & 0 & $20.30(3.82)$ & $18-56$ \\
\hline & College 2 & 113 & 51 & 59 & 3 & $19.76(1.02)$ & $18-23$ \\
\hline \multirow[t]{2}{*}{ Korea } & College & 118 & 71 & 47 & 0 & $22.85(2.37)$ & $18-29$ \\
\hline & Panel & 174 & 85 & 89 & 0 & $39.22(11.11)$ & $18-59$ \\
\hline India & Mturk & 553 & 399 & 154 & 0 & $32.91(8.96)$ & $20-78$ \\
\hline Norway & College & 125 & 32 & 93 & 0 & $28.53(10.99)$ & $18-61$ \\
\hline Singapore & College & 104 & 40 & 63 & 1 & $21.58(1.74)$ & $18-26$ \\
\hline Portugal & College & 258 & 71 & 180 & 7 & $23.16(7.35)$ & $18-89$ \\
\hline Angola & College & 150 & 48 & 91 & 11 & $23.72(5.49)$ & $18-64$ \\
\hline Germany & College & 155 & 28 & 122 & 5 & $25.40(10.23)$ & $18-80$ \\
\hline
\end{tabular}


To this end, we conducted multi-group confirmatory factor analyses (MG-CFA) and tested configural and metric measurement invariances using Mplus 7.4 (Muthén \& Muthén, 1998-2006). Configural invariance is supported when the number of latent factors (happy, psychologically rich, and meaningful life) and the number of items on each factor are the same across nations. Metric invariance is supported when each item's factor loading on the factor is equivalent across nations. Our criteria for evaluating model fit were $C F I$ close to .95 and RMSEA below .15 given that more liberal cutoffs can be employed when there are more than 10 groups to be compared (Rutkowski \& Svetina, 2014). Accordingly, for model fit comparison, we used more liberal criteria of $\triangle C F I \leq .020$ and $\triangle R M S E A \leq .030$ for evaluating metric invariance. Prior to analyses, we excluded 37 responses from the Angola sample due to excessive missing data (i.e., missing more than 8 of the 15 items). Because we had multiple samples within a nation, we first conducted a MG-CFA for 15 items with the 13 samples. The configural invariance for the three-factor model did not fit the data well with these 15 items: $C F I=.810$, $T L I=.771, R M S E A=.12190 \%$ CI $[.118, .125]$, $S R M R=.103, \chi^{2}(1131)=5125.452, p<.001$. Thus, we inspected items whose $R$ square was less than .30 in any of the samples and removed the following 6 items: stable, simple, dramatic, rich, virtuous, and devotion. This left three items remaining for each of the three types of the good life (the happy life: "comfortable," "happy," and "pleasant"; the psychologically rich life: "eventful," "interesting," and "full of surprise"; the meaningful life: "meaningful," "fulfilling," and "sense of purpose"). We tested configural invariance using these 9 items across the 13 samples and found that model fit was good: $C F I=.947, T L I=.921, R M S E A=.09390 \% C I$ $[.087, .100], S R M R=.059, \chi^{2}(312)=956.036, p<.001$.

Next, because we were interested in looking at patterns of an ideal life at the country level, we examined measurement invariance in the subsamples within a nation (three subsamples in the USA, two subsamples in Japan and Korea, respectively) using the remaining 9 items.

Overall, configural measurement invariance was supported for all three nations: $C F I=.958, T L I=.936, R M S E A=.084$ $90 \%$ CI [.074, .094], SRMR $=.053, \chi^{2}(72)=288.400$, $p<.001$ for the USA; $C F I=.931, T L I=.897, R M S E A=.117$ $90 \% C I[.092, .142], S R M R=.079, \chi^{2}(48)=123.980$, $p<.001$ for Japan; $C F I=.961, T L I=.942, R M S E A=.071$ $90 \%$ CI $[.044, .096], S R M R=.068, \chi^{2}(48)=82.979$, $p=.001$ for Korea. Thus, we collapsed the subsamples in each nation.

How did people describe their ideal lives? The patterns of self-rated characteristics, as seen in Table 2, show that in every country except for Norway and Germany, participants' selfdescribed ideal lives were best characterized as a happy life, followed by a meaningful life. In all 9 countries, participants' spontaneous descriptions of their ideal life were least characterized as a psychologically rich life, of the three dimensions. Nevertheless, many of those ideal lives were rich: average ratings of richness ranged from 3.7 to 5.62 , far above the bottom of the scale ( $1=$ "not at all" rich). Indeed, in all but Japan and Korea, mean ratings for a psychologically rich life were markedly above the midpoint (4). Thus, what participants described as an ideal life was characterized not only as a happy life (happy, comfortable, pleasant) and a meaningful life (meaningful, fulfilling, sense of purpose), but also, albeit to a lesser extent, as a psychologically rich life (eventful, interesting, full of surprise).

As seen in Table 3, idealizing a psychologically rich life was unrelated or only weakly related to idealizing a happy life in most countries, with the exception of Angola and India $(r \mathrm{~s}=-.11$ to .51 , median $r=.17)$. It was moderately positively associated with idealizing a meaningful life in all 9 countries $(r \mathrm{~s}=.34-.59$, median $r=.43)$. Idealizing a happy life was likewise positively correlated with idealizing a meaningful life ( $r \mathrm{~s}=.22$ to .68 , median $r=.41$ ). That is, individuals who idealize a meaningful life tend also to idealize a happy life and a psychologically rich life. In contrast, individuals who idealize a psychologically rich life do not necessarily idealize a happy life (and vice versa).

Next, we explored whether people's current levels of hedonic and eudaimonic well-being were systematically associated with their ideal life. It is possible, for instance, that individuals who are not currently satisfied with their lives idealize a happy life (the compensatory hypothesis), whereas those who are already satisfied with their lives and find their lives to be meaningful tend to idealize a psychologically rich life (the stage hypothesis). Conversely, the opposite possibility could be true: individuals who are already satisfied with their lives might value a happy life, whereas those who find their lives to be meaningful might value a meaningful life (the justification hypothesis).

We did not find any evidence in support of the compensatory hypothesis. Idealizing a psychologically rich life was only weakly related to self-reports of life satisfaction $(r=-.04$ to .28 , median $r=.11$ ), positive affect ( $r=.08$ to .38 , median $r=.18)$, negative affect ( $r=-.13$ to .16 , median $r=-.04)$, and the presence of meaning in life $(r=-.05$ to .37 , median $r=.17)$. Similarly, idealizing a happy life was weakly associated with self-reports of life satisfaction $(r=-.06$ to .24 , median $r=.07$ ), positive affect ( $r=-.00$ to .37 , median $r=.17$ ), negative affect ( $r=-.25$ to .06 , median $r=-.07)$, and meaning in life ( $r=.01$ to .30 , median $r=.11)$. Idealizing a meaningful life was also weakly correlated with self-reports of life satisfaction $(r=-.02$ to .25 , median $r=.16$ ), positive affect ( $r=-.07$ to .36 , median $r=.17$ ), negative affect $(r=-.27$ to .19 , median $r=-.07$ ), but consistently correlated with the presence of meaning in life ( $r=.13$ to .40 , median $r=.29$ ). In sum, the type of good life that people valued was relatively independent of their current levels of hedonic and eudaimonic 
Table 2 Percent chosen in the forced choice of a happy life, a psychologically rich life, and a meaningful life for each nation and mean $(S D)$ of self-rated characteristics ratings

\begin{tabular}{|c|c|c|c|c|c|c|}
\hline & \multicolumn{2}{|l|}{ Happy life } & \multicolumn{2}{|c|}{ Psychologically rich life } & \multicolumn{2}{|c|}{ Meaningful life } \\
\hline & $M(S D)$ & $\%$ & $M(S D)$ & $\%$ & $M(S D)$ & $\%$ \\
\hline USA & $6.48(0.76)$ & 62.2 & $4.98(1.30)$ & 13.2 & $6.23(0.99)$ & 24.7 \\
\hline Germany & $6.04(0.96)$ & 49.7 & $5.19(1.25)$ & 16.8 & $6.25(1.02)$ & 33.5 \\
\hline Norway & $5.39(1.18)$ & 50.8 & $4.99(1.14)$ & 15.3 & $5.52(1.06)$ & 33.9 \\
\hline Portugal & $6.39(0.76)$ & 51.2 & $5.62(1.10)$ & 15.1 & $6.32(0.87)$ & 33.7 \\
\hline Japan & $6.18(1.02)$ & 65.5 & $3.70(1.51)$ & 15.5 & $5.26(1.35)$ & 19.0 \\
\hline Korea & $5.99(0.88)$ & 69.9 & $3.93(1.28)$ & 15.8 & $5.59(1.03)$ & 14.4 \\
\hline Singapore & $6.33(0.80)$ & 54.8 & $4.69(1.33)$ & 6.7 & $6.03(1.11)$ & 38.5 \\
\hline India & $6.13(0.95)$ & 55.5 & $5.55(1.09)$ & 16.1 & $5.90(1.01)$ & 28.4 \\
\hline Angola & $5.82(1.15)$ & 54.3 & $5.18(1.43)$ & 9.3 & $5.66(1.30)$ & 36.4 \\
\hline
\end{tabular}

Characteristics ratings were made on the 1 (not at all)-7 (very much)-point scale well-being. Overall, this pattern of the results shows no support for the compensatory hypothesis, very weak support for the stage hypothesis, and some support for the justification hypothesis, for a meaningful life.

\section{What Is an Ideal Life? Forced Choice}

Because all three forms of a good life are normatively desirable, it is difficult to tell which one is more desirable than others. In addition, cultural differences in response style (e.g., greater tendency to use the neutral point among East Asians; Chen, Lee, \& Stevenson, 1995) could obscure cultural variations in a 7-point scale. Thus, next we analyzed forced choice data: what people selected as their ideal life when they could pick only one of the three options. As seen in Table 2, in every sample, the majority favored a happy life overall (49.7 to $69.9 \%$ ). In all but one sample (Korea), the second most popular choice was a meaningful life (14.2 to $38.5 \%$ ). Finally, while a psychologically rich life was least frequently

Table 3 Correlations between three types of ideal lives in study 1

\begin{tabular}{llll}
\hline & $\begin{array}{l}\text { Rich life and } \\
\text { happy life }\end{array}$ & $\begin{array}{l}\text { Rich life and } \\
\text { meaningful life }\end{array}$ & $\begin{array}{l}\text { Happy life and } \\
\text { meaningful life }\end{array}$ \\
\hline USA & $.09 * *$ & $.37 * * *$ & $.41 * * *$ \\
Germany & $.23 * *$ & $.47 * * *$ & $.49 * * *$ \\
Norway & .17 & $.34 * * *$ & $.45 * * *$ \\
Portugal & $.26 * * *$ & $.36 * * *$ & $.35 * * *$ \\
Japan & -.11 & $.43 * * *$ & $.33 * * *$ \\
Korea & .06 & $.34 * * *$ & $.22 * * *$ \\
Singapore & .05 & $.56 * * *$ & $.23 *$ \\
India & $.51 * * *$ & $.59 * * *$ & $.68 * * *$ \\
Angola & $.51 * * *$ & $.55^{* * * *}$ & $.53 * * *$ \\
\hline
\end{tabular}

$* p<.05, * * p<.01, * * * p<.001$ chosen, a non-trivial number of participants still selected it as their ideal life, ranging from 6.7 to $16.8 \%$ of the participants. These findings suggest that a psychologically rich life is one type of a good life and is actively favored even over a happy life or a meaningful life by a minority of individuals across a diverse set of cultures.

Were people's forced choice selections related to their current levels of hedonic and eudaimonic well-being? To find out, we conducted the same analyses with people's forcedchoice selections as we did with their self-rated ideal life. As seen in Table 4, there were no differences among the three choices in any measures of hedonic and eudaimonic wellbeing measures in Angola, Germany, Japan, Korea, and Portugal. Among Americans, individuals who idealized a psychologically rich life reported their lives were less meaningful than those who idealized a happy life or a meaningful life. Among Singaporeans, those who idealized a happy life reported their lives were less meaningful than those who idealized a meaningful life. Among Norwegians, those who idealized a psychologically rich life reported their lives were less meaningful than those who idealized a happy life. And, finally, among Indians, those who idealized a meaningful life reported less negative affect and more meaning than those who idealized a happy life.

In sum, the results of study 1 suggest that a non-trivial number of participants selected a psychologically rich life as their ideal life, ranging from 7 to $17 \%$, and that most people's self-described ideal lives were relatively rich. People's choice of an ideal life, on the other hand, was not systematically or reliably associated with their current levels of hedonic or eudaimonic well-being. However, it is unclear the extent to which people can self-report on their ideal life or know what they want. It is possible that, while people may indeed desire a psychologically rich life, they may not think of it in such terms or have a schema for psychological richness in the same way that they do for happiness or meaning. Thus, an alternative to 
asking people about their ideal lives directly is to investigate the question indirectly - namely, by asking what they most regret in life and inferring (from their answer) how their lives would be different if the incident had not occurred.

\section{Study 2a: What Do You Regret?}

Just as it is important to assess both avoidance and approach goals to understand people's goals and desires in the moment (Elliot, 1999), it is imperative to explore what people wish they had avoided in their lives, to understand fully what they wish their lives might have been (cf., possible and feared selves, Markus \& Nurius, 1986). Thus, whereas in study 1 we encouraged respondents to think of the type of life they desire and actively approach, in study 2 , we first asked participants what they regret most in their lives. We then asked whether undoing or reversing this regrettable life event would have made their lives happier, more meaningful, or psychologically richer. Regret provides a unique window through which we could observe the events that people wish never happened and the type of life events that they are trying to avoid in the future (King \& Hicks, 2007; Saffrey, Summerville, \& Roese, 2008; Zeelenberg, 1999). It also allows us to infer, indirectly, how they wish their lives were different today-namely, whether they wish they were leading happier, more meaningful, or richer lives.

\section{Method}

\section{Participants}

Participants were 2157 Americans recruited from a large online sampling panel. Out of the 2157 who started the survey, 1733 completed the survey. Of those who completed the survey, 1624 (93.7\%) passed the attention check items, and 1611 (92.9\%) both passed the attention check and completed the regret items that served as our critical dependent measure.

\section{Procedure and Materials}

Participants completed a survey in May 2018 that examined the replicability of past psychological findings. Toward the end of the survey, we included an open-ended item regarding the participants' greatest regret in life. Then, we asked if they could theoretically undo the event, whether their lives would have been happier, more meaningful, or psychologically richer (forced choice).

\section{Results}

Reversing your biggest regret in life should, presumably, result in a better life. But what kind of a better life? Of the 1611 
people we asked, answers were almost equally split: $35.7 \%$ (575) said that undoing the regretful event would have made their lives happier, $36.7 \%$ (592) said that it would have made their lives more meaningful, and the remaining $27.6 \%$ (444) said that it would have made their lives psychologically richer. For instance, one respondent wrote that they regretted "not going to a 4-year college to get a degree. I feel like I missed out on some interesting experiences," and indicated that if they could undo this event, their life would have been psychologically richer.

There were very small but significant age differences in people's reports of how their lives would be affected by undoing their biggest regret among the three choices, $F(2$, $1624)=3.26, p=.038$. Older participants were slightly more likely to report that undoing their biggest regret would have made their lives richer, while younger participants were slightly more likely to report that it would made their life happier according to post hoc tests, $p=.042, d=.15$.

Participants who said undoing the regretful event would have made their lives happier were 44.34 years old, on average $(S D=11.71)$, versus 46.10 years $(S D=$ 10.80) for those who said undoing it would have made their lives psychologically richer. In contrast, people who said undoing it would have made their lives more meaningful were in the middle, at 45.50 years on average $(S D=11.17)$. One question is whether psychological richness is more pertinent for some groups of people than others. We found very little evidence to support gender or ethnic differences in whose lives would have become richer as a result of undoing their life's biggest regret (see supplemental materials).

In sum, using a counterfactual method, we found that roughly $28 \%$ of American adults desired a psychologically rich life, far higher than the number who reported desiring so explicitly. But to what extent is this the case across other cultures?

\section{Study 2b}

In study $2 \mathrm{a}$, we found that almost a third of Americans wished they were living a richer life, when this question was assessed indirectly, compared to only $13.2 \%$ when asked explicitly. We conducted a second study (study $2 b)$ to see if we could replicate these US findings in Korea for two reasons: (1) because Koreans were less likely than others to explicitly say that they value a psychologically rich life in study 1 and (2) Korea is culturally very distinct of the USA, both in terms of individualismcollectivism (Hofstede, 2001) and tightness-looseness (Gelfand et al., 2011).

\section{Method}

\section{Participants}

Participants were 680 Koreans (338 males, 342 females) recruited from a large online sampling panel. The age of the participants ranged from 19 to $69(M=44.26, S D=13.08)$. Participants completed a survey in January 2020. As part of the survey, we included an open-ended item regarding the participants' greatest regret in life. Then, we asked if they could theoretically undo that event, whether their lives would have been happier, more meaningful, or psychologically richer (forced choice).

\section{Results}

Similar to the US findings, $27.6 \%$ (188) of Korean participants said that undoing the regretful event would have made their lives happier, $37.4 \%$ (254) said that it would have made their lives more meaningful, and the remaining 35\% (238) said that it would have made their lives psychologically richer.

There were no significant age differences in these choices, $F(2,677)=2.41, p=.091$. Replicating study 2a, older participants were slightly more likely to report that undoing their biggest regret would have made their lives richer $\left(M_{\mathrm{age}}=\right.$ $45.64, S D=12.72$ ), while younger participants were slightly more likely to report that it would made their life happier $\left(M_{\text {age }}=42.90, S D=12.80\right), d=.21$. In contrast, people who said undoing it would have made their lives more meaningful were in the middle, at 43.97 years on average $(S D=13.55)$. However, these differences were not conventionally significant.

In sum, we replicated the main findings from Americans in study 2a among Koreans in study 2b: $35 \%$ of Korean participants said that their lives would have been psychologically richer if they could redo or undo their most regretted life event, compared to just $15.8 \%$ who reported desiring a psychologically rich life when asked explicitly. These rates were very similar to those found among Americans in study 2a, suggesting that people may very well desire a psychologically richer life, even when they do not report idealizing psychological richness when asked explicitly.

\section{General Discussion}

Recent research has found that a psychologically rich life is distinct from a happy or meaningful life in terms of personality predictors (Oishi et al., 2019), life experiences (Oishi, Choi, Heintzelman, et al., 2020), and political orientations Oishi, Westgate, Heintzelman, et al., 2020). We conducted the current research with the goal of investigating whether some people desire a psychologically rich life more so than 
two well-established ideal lives: a happy life and a meaningful life.

In study 1 (a 9-nation study), we found that most people's self-described ideal lives were psychologically rich. When forced to choose, however, the majority favored a happy life (49.7 to $69.9 \%$ ) or a meaningful life (14.2 to $38.5 \%$ ). Even so, a substantial minority of participants still favored a psychologically rich life, even at the expense of a happy life or a meaningful life, ranging from $6.7 \%$ (Singapore) to $16.8 \%$ of participants (Germany). In studies $2 \mathrm{a}$ and $2 \mathrm{~b}$, we found that these numbers were even higher when desire for a psychologically rich life was measured indirectly. Roughly $28 \%$ of Americans and $35 \%$ of Koreans reported that their lives would have been psychologically richer, if they could undo the most regretted event of their lives. These data suggest that most people's ideal lives are not just happy or meaningful but also psychologically rich, and that when forced to pick one, a nontrivial number of people desire a rich life more than a happy or a meaningful life. When measured indirectly, just as many people wish their lives were richer as do wish their lives were happier or more meaningful.

As discussed above, well-being research has been dominated by two concepts: hedonic and eudaimonic well-being (Diener et al., 1999; Vittersø, 2016). Our present research suggests a broader view. Namely, that a psychologically rich life is another type of a good life that some individuals lead and desire, and one that is not captured by current empirical conceptions of a good life. Importantly, unlike happiness or meaning, our conception of richness includes moments of discomfort and unpleasant emotion. Understanding that a good life may not always be pleasant or sacrificial - that there is value to individuals in leading lives that investigate truth, knowledge, and deep encounters with the world around them - may help us understand why people sometimes seek out such experiences (e.g., studying abroad, reading James Joyce's Ulysses) at the expense of their own comfort and security. The ability to make sense of such behaviors is a benefit of conceptualizing a psychologically rich life as another type of a good life that people value and seek out.

Indeed, people with psychologically rich lives experience both positive and negative emotions more intensely, whereas those leading happy or meaningful lives experience positive emotions more intensely but negative emotions less intensely (Oishi, Westgate, Heintzelman, et al., 2020). It will be fruitful to explore how a psychologically rich life is associated with other important dimensions of emotional experiences such as the diversity of emotional experiences (Quoidbach et al., 2014), affect valuation (Tsai, 2007), and emotion differentiation and regulation (Barrett, Gross, Christensen, \& Benvenuto, 2001).

Importantly, although we tested the relative importance of three types of the ideal life, we do not claim there cannot be others. Based on Schwartz's (1992) value theory, Tamir and colleagues (Tamir et al., 2016; Tamir, Schwartz, Oishi, \& Kim, 2017) recently examined four types of desired and experienced emotions: self-transcendence (e.g., love), selfenhancement (e.g., pride), openness (e.g., interest), and conservation (e.g., contentment). A psychologically rich life corresponds well to openness-related emotions, whereas a happy life corresponds well to conservation and self-enhancement emotions; a meaningful life appears to match well with selftranscendent emotions. Given that the literature suggests that there are at least 11 universal human values (Schwartz, 1992), and that values are guiding principles in life, there may be many more than the three ideal lives presented here.

We also acknowledge the limitations of the current research. Most critically, we did not examine the potential consequences of leading a psychologically rich life. It is crucial to test whether the consequences of a psychologically rich life are indeed different from a happy or meaningful life-and whether these consequences are a good thing. Second, while study 1 included diverse cross-cultural samples, study 2 was limited to the USA and Korea. It is important to explore what it means to live a psychologically rich life (and whether doing so is desirable) in other non-Western, non-democratic, relatively poor societies.

\section{Conclusion}

Well-being researchers have long debated the merits of hedonic and eudaimonic well-being. It is time to move beyond this dichotomous model of well-being. Hesse (1932)/1984) in his classic novel, writing from Narcissus' viewpoint, wondered whether the type of life that Goldmund led - eventful, dramatic, and interesting - was also a good life. We found that many people believe it is - that a small but consistent minority of people across cultures not only view such a life as a good life but prefer it to a happy or meaningful life, and that a third of people report that undoing or reversing their biggest regret in life would have made their lives not happier, and not more meaningful — but psychologically richer instead. By considering the concept of a psychologically rich life, those peoplelike their literary counterpart Goldmund - are no longer lost to science. As such, we believe that taking the psychologically rich life seriously will deepen, broaden, and, yes, enrichen our understanding of well-being.

\section{Additional Information}

Acknowledgments We thank JP Bouvet and TJ Gill for their help with editing the manuscript. 
Funding Information This research was supported by a grant from the John Templeton Foundation and Saint Louis University to Shigehiro Oishi and Lorraine L. Besser (G011993).

Data Availability All materials and data can be found at https://osf.io/ yuf92/.

Conflict of Interest The authors declare that there are no conflicts of interest.

Ethical Approval Approval was obtained from the ethics committee of University of Virginia. The procedures used in this study adhere to thetenets of the Declaration of Helsinki.

Informed Consent Informed consent was obtained from all individual participants included in the study.

\section{References}

Barrett, L. F., Gross, J., Christensen, T. C., \& Benvenuto, M. (2001). Knowing what you're feeling and knowing what to do about it: mapping the relation between emotion differentiation and emotion regulation. Cognition and Emotion, 15, 713-724.

Besser, L., \& Oishi, S. (in press). The psychologically rich life. Philosophical Psychology.

Chen, C., Lee, S.-y., \& Stevenson, H. W. (1995). Response style and cross-cultural comparisons of rating scales among East Asian and North American students. Psychological Science, 6, 170-175.

Diener, E. (2000). Subjective well-being: the science of happiness and a proposal for a national index. American Psychologist, 55, 34- 43.

Diener, E., Emmons, R. A., Larsen, R. L., \& Griffin, S. (1985). The satisfaction with life scale. Journal of Personality Assessment, 49, $71-75$.

Diener, E., Suh, E. M., Lucas, R. E., \& Smith, H. (1999). Subjective wellbeing: three decades of progress. Psychological Bulletin, 125, 276 302.

Diener, E., Wirtz, D., Tov, W., Kim-Prieto, C., Choi, D., Oishi, S., \& Biswas-Diener, R. (2010). New well-being measures: short scales to assess flourishing and positive and negative feelings. Social Indicators Research, 97, 143-156.

Elliot, A. J. (1999). Approach and avoidance motivation and achievement goals. Educational Psychologist, 34, 169-189.

Ford, B. Q., Lwi, S., Hankin, B., Gentzler, A., \& Mauss, I. B. (2018). The cost of believing emotions are uncontrollable: youths' beliefs about emotion predict emotion regulation and depressive symptoms. Journal of Experimental Psychology: General, 47, 1170-1190.

Gelfand, M. J., Raver, J. L., Nishii, L., Leslie, L. A., Lun, J., Lim, B. C., et al. (2011). Differences between tight and loose cultures: a 33nation study. Science, 332, 1100-1104.

Gruber, J., Mauss, I. B., \& Tamir, M. (2011). A dark side of happiness? How, when, and why happiness is not always good. Perspectives on Psychological Science, 6(3), 222-233.

Heintzelman, S. J., \& King, L. A. (2014). Life is pretty meaningful. American Psychologist, 69, 561-574.

Henrich, J., Heine, S. J., \& Norenzayan, A. (2010). The weirdest people in the world? Behavioral and Brain Sciences, 33, 61-83.

Hesse, H. (1932/1984). Narcissus and Goldmund. New York: Bantam.

Hofstede, G. (2001). Culture's consequences: comparing values, behaviors, institutions and organizations across nations. Thousand Oaks, CA: Sage.
King, L. A., \& Hicks, J. A. (2007). Whatever happened to "what might have been?" Regrets, happiness, and maturity. American Psychologist, 62, 625-636.

Markus, H. R., \& Nurius, P. (1986). Possible selves. American Psychologist, 41, 954-969.

Muthén, L. K., \&Muthén, B. O. (1998-2006). Mplus user's guide. (4th edn). Los Angeles, CA: Muthén \& Muthén.

Oishi, S., \& Choi, H. (2020). What is a psychologically rich experience? An exploration of visual images. A paper under review.

Oishi, S., Choi, H., Buttrick, N., Heintzelman, S. J., Kushlev, K., Westgate, E. C., Tucker, J., Ebersole, C. R., Axt, J., Gilbert, E., Ng, B. W., \& Besser, L. L. (2019). The psychologically rich life questionnaire. Journal of Research in Personality, 81, 257-270.

Oishi, S., Choi, H., Heintzelman, S. J., Westgate, E. C., Buttrick, N., Ebersole, C. R., \& Besser, L. L. (2020). The triad model of a good life: a happy life, a meaningful life, and a psychologically rich life. A paper in preparation.

Oishi, S., Choi, H., Liu, A., \& Kurtz, J. (2020). Experiences associated with psychological richness. A paper under review.

Oishi, S., Westgate, E., Heintzelman, S. J., Buttrick, N., Choi, H., \& Cha, Y. (2020). The politics and curiosity of a happy life, a meaningful life, and a psychologically rich life. A paper in preparation.

Quoidbach, J., Gruber, J., Mikolajczak, M., Kogan, A., Kotsou, I., \& Norton, M. I. (2014). Emodiversity and the emotional ecosystem. Journal of Experimental Psychology: General, 143, 2057-2066.

Rutkowski, L., \& Svetina, D. (2014). Assessing the hypothesis of measurement invariance in the context of large-scale international surveys. Educational and Psychological Measurement, 74, 31-57.

Ryff, C. D. (1989). Happiness is everything, or is it? Exploration on the meaning of psychological well-being. Journal of Personality and Social Psychology, 57, 1069-1081.

Saffrey, C., Summerville, A., \& Roese, N. J. (2008). Praise for regret: people value regret above other negative emotions. Motivation and Emotion, 32, 46-54.

Schwartz, S. H. (1992). Universals in the content and structure of values: theoretical advances and empirical tests in 20 countries. Advances in Experimental Social Psychology, 25, 1-65.

Steger, M. F., Frazier, P., Oishi, S., \& Kaler, M. (2006). The Meaning in Life Questionnaire: assessing the presence of and search for meaning in life. Journal of Counseling Psychology, 53, 80-93.

Tamir, M., Schwartz, S. H., Cieciuch, J., Riediger, M., Torres, C., Scollon, C., Dzokoto, V., Zhou, X., \& Vishkin, A. (2016). Desired emotions across cultures: a value-based account. Journal of Personality and Social Psychology, 111, 67-82.

Tamir, M., Schwartz, S. H., Oishi, S., \& Kim, M. (2017). The secret to happiness: feeling good or feeling right? Journal of Experimental Psychology: General, 146, 1448-1459.

Tsai, J. (2007). Ideal affect: cultural causes and behavioral consequences. Perspectives on Psychological Science, 2, 242-259.

Vittersø, J. (2016). Handbook of eudaimonic well-being. Cham, Switzerland: Springer.

Westgate, E.C., \& Losee, J. (2020). Mere categorization: the process of categorization is inherently rewarding. Manuscript in preparation.

Westgate, E. C., \& Wilson, T. D. (2018). Boring thoughts and bored minds: the MAC model of boredom and cognitive engagement. Psychological Review, 125, 689-713.

Zeelenberg, M. (1999). The use of crying over spilled milk: a note on the rationality and functionality of regret. Philosophical Psychology, 12, $325-340$ 